\title{
Characteristics and outcomes of preterm neonates according to number of doses of surfactant received
}

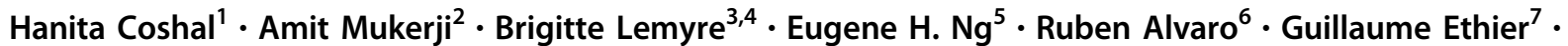 \\ Eugene W. Yoon ${ }^{1} \cdot$ Marc Beltempo ${ }^{8,9} \cdot$ Prakesh S. Shah $\mathbb{1}^{1,10,11}$
}

Received: 29 April 2020 / Revised: 16 July 2020 / Accepted: 3 August 2020 / Published online: 13 August 2020

(C) The Author(s), under exclusive licence to Springer Nature America, Inc. 2020

\begin{abstract}
Objectives To identify characteristics and outcomes of infants who received multiple doses of surfactant vs those who received one dose or none.

Study design In this retrospective study, we included neonates of 22-28 weeks' gestation admitted to NICUs in the Canadian Neonatal Network. Patients were divided into three groups: no surfactant, single dose, and multiple doses. The primary outcome was a composite of mortality or any of the major morbidities, including severe neurological injury, bronchopulmonary dysplasia, or $\geq$ stage 3 retinopathy of prematurity.

Results Of 8024 eligible neonates, 2461 (31\%) did not receive surfactant, 3545 (44\%) received one dose, and $2018(25 \%)$ received $>1$ dose. Receiving one or more doses of surfactant was associated with significantly higher adjusted odds of mortality or major morbidities in a dose-dependent manner.

Conclusions Receiving one or more doses of surfactant was associated with adverse neonatal outcomes. Receipt of more than one dose may reflect underlying severe lung immaturity.
\end{abstract}

\section{Introduction}

Surfactant replacement therapy has been the cornerstone of medical management for extremely preterm neonates. A majority of infants in this gestational age (GA) group receive surfactant therapy to treat or prevent respiratory distress

Supplementary information The online version of this article (https:// doi.org/10.1038/s41372-020-00779-9) contains supplementary material, which is available to authorized users.

Prakesh S. Shah

Prakeshkumar.Shah@sinaihealth.ca

1 Maternal-Infant Care Research Centre, Mount Sinai Hospital, Toronto, ON, Canada

2 Department of Pediatrics, McMaster Children's Hospital, Hamilton, ON, Canada

3 Division of Neonatology, Children's Hospital of Eastern Ontario, Ottawa, ON, Canada

4 Division of Neonatology, The Ottawa Hospital, Ottawa, ON, Canada

5 Department of Newborn \& Developmental Paediatrics, Sunnybrook Health Sciences Centre, Toronto, ON, Canada syndrome (RDS) [1]. Usually, neonates respond to one dose of surfactant and are either extubated or stabilized using lower oxygen requirements [2] and lower mean airway pressure support. A single dose of surfactant in a randomized controlled trial was not associated with reduction in mortality but only reduced severity of RDS and pneumothorax [3]. However, the respiratory condition worsens in some neonates after $\mathrm{a}$ few $\mathrm{h}$ to the presurfactant state and they require additional doses of surfactant [2]. In randomized controlled trials of surfactant therapy, the incidence of administration of repeat

6 Department of Pediatrics and Child Health, University of Manitoba, Winnipeg, MB, Canada

7 Department of Nursing, CHU Sainte-Justine, Montreal, QC, Canada

8 Department of Pediatrics, McGill University Health Centre, Montreal, QC, Canada

9 Department of Pediatrics, Montreal Children's Hospital, Montreal, QC, Canada

10 Department of Pediatrics, Mount Sinai Hospital, Toronto, ON, Canada

11 Department of Pediatrics and Institute of Health Policy, Management and Evaluation, University of Toronto, Toronto, ON, Canada 
doses of surfactant has varied from 20 to $50 \%$ [4]. The effect of surfactant in reducing mortality was only shown in studies that tested multiple doses.

The practice of surfactant treatment has evolved over years from administration. It started to be used after a neonate exceeded a certain cut-off for respiratory support, then changed to prophylactic administration irrespective of clinical status immediately after birth [5], early vs delayed administration [6], followed by the use of continuous positive airway pressure (CPAP) to establish functional residual capacity and give surfactant only if needed [7], and more recently, to either immediate extubation after surfactant [8] or administration of surfactant via thin catheters [9]. Even with these different approaches, a certain number of neonates need or receive repeat doses of surfactant. Data on the characteristics and clinical outcomes of neonates who receive multiple surfactant replacement treatments are scant. In a previous study, those who received $>1$ dose were of lower GA $(28.9 \pm 2.2$ weeks vs $29.2 \pm 2.5$ weeks $)$ and lower birth weight $(\mathrm{BW})(1189 \pm 321 \mathrm{~g}$ vs $1218 \pm 327 \mathrm{~g})$ compared to neonates who improved after one dose of surfactant [10]. Lack of antenatal steroids [11] and presence of infection were identified as risk factors for receiving multiple doses of surfactant. Group B Streptococcal infection [12], maternal chorioamnionitis [2], atelectasis, pulmonary hemorrhage, and asphyxia $[13,14]$ are the reasons postulated for surfactant inactivation [11]. On the other hand, Hoekstra et al. [15] reported that multiple doses of surfactant led to higher survival in very preterm neonates with RDS. In a randomized trial of neonates 30-36 weeks' gestation with RDS, Dunn et al. [16] concluded that those who received multiple doses of surfactant were more stable and easily manageable following treatment due to the reduced severity of RDS. Thus, the literature is limited and conflicted on the effect of multiple doses of surfactant on neonatal outcomes. A majority of studies were conducted in an era of relatively liberal use of surfactant and high rates of intubation and ventilation. In the recent era of higher rates of CPAP use and either selective use or less invasive administration of surfactant [17], repeated surfactant use has not been evaluated. In the present study, our objective was to identify characteristics and report on outcomes of preterm neonates $<29$ weeks' gestation from a large, national cohort who received multiple doses of surfactant vs those who received one dose of surfactant or no surfactant.

\section{Patients and methods}

\section{Setting and eligibility criteria}

The Canadian Neonatal Network (CNN) is a collaborative research platform of all 31 tertiary neonatal intensive care units (NICUs) in the country. The network's database contains medical data on infants and mothers admitted to these NICUs, which was used for this study. Infants born at $<29$ weeks' gestation and admitted between January 1, 2014 and December 31, 2018 were included. Infants were divided into three groups based on surfactant dosage: no surfactant, single dose, and multiple doses. Infants with major congenital anomalies or moribund at admission for whom palliative care was provided immediately after birth were excluded from the study.

\section{Data collection}

The data used in this study were collected from patient charts at the individual sites by trained abstractors using the CNN Abstractor's Manual [18]. The data were entered electronically and transmitted to the central coordinating center. The CNN database has been shown to have very high reliability and internal consistency [19]. Ethics approvals for this study were received from the Research Ethics Board at Mount Sinai Hospital and the Executive Committee of the CNN.

\section{Exposure (surfactant doses)}

Surfactant administration at individual units followed unit protocol or the attending team's discretion based on clinical signs and support requirements. We did not have data available on what triggered surfactant administration in an individual neonate, and we did not have $\mathrm{x}$-ray information suggestive of surfactant deficiency. The types of surfactant used and dosages delivered varied across Canadian NICUs. Surfactants included bovine lipid surfactant (BLES Biochemicals Inc., London, ON, Canada), beractant (Survanta, Abbvie Inc., Chicago, IL, USA), and poractant alfa (Curosurf, Chiesi USA Inc., Cary, NC, USA). Surfactant therapy administration was according to recommendations made by the Canadian Pediatric Society [20] to ensure safety and accuracy. These recommendations suggest administration of surfactant to intubated patients with RDS (grade A evidence), prophylactic administration after stabilization in intubated preterm neonates at significant risk of RDS (grade A evidence), repeat administration to neonates who have persistent or recurrent need for oxygen within the first $72 \mathrm{~h}$ of age (grade A evidence), and retreatment to be considered when oxygen requirement is $>30 \%$ within $2-6 \mathrm{~h}$ after administration of the first dose (grade A evidence). Patients were divided into three groups: no surfactant, single dose, and multiple doses. The majority of surfactants were administered in the endotracheal tube; however, during the latter part of the study period, some units were attempting less invasive methods of surfactant administration. We did not have data to differentiate the method of administration. 


\section{Outcomes}

The primary outcome was a composite of mortality prior to discharge or major morbidity. Major morbidities included severe neurological injury, bronchopulmonary dysplasia (BPD), and severe retinopathy of prematurity (ROP). Severe neurological injury was defined as the presence of grade 3 or 4 intraventricular hemorrhage or persistent periventricular echogenicity on head ultrasound [21]. BPD was defined as the receipt of any respiratory support at 36 weeks' postmenstrual age or at the time of discharge to a level 2 hospital [18]. Severe ROP was defined as stage 3 or higher retinopathy or treated retinopathy (laser or injection therapy) in either eye. The secondary outcomes were the individual components of the composite outcome. We also evaluated mortality or BPD as an outcome similar to that reported in multiple trials of surfactant.

\section{Definitions}

GA was defined as the best estimate based on the date of in vitro fertilization, early ultrasound, last menstrual period, obstetric estimate, or pediatric estimate, in that hierarchical order. Small for GA (SGA) was defined as BW less than the 10th percentile for GA and sex. Extensive cardiopulmonary resuscitation was defined as receipt of chest compression or epinephrine within the first $30 \mathrm{~min}$ of birth. Data on maternal and infant characteristics and other predisposing factors were retrieved from the database for analysis.

\section{Statistical analyses}

The maternal and infant characteristics were compared among the three groups using the Chi-square test for categorical variables and the $F$-test in an ANOVA analysis for continuous variables. A multivariable logistic regression model was used to evaluate the associations of the exposures and the outcomes and adjust for patient characteristics that differed in the descriptive statistics $(P<0.05)$. Generalized estimating equation was applied in each regression model to account for correlations within the same site and within multiple births. The group of neonates who did not receive surfactant was the reference group. We also compared neonates who received one dose of surfactant with neonates who received $>1$ dose. Subgroup analyses were conducted for neonates of 22-25 weeks' GA and 26-28 weeks' GA. A $P$ value of $<0.05$ was considered statistically significant.

\section{Results}

A total of 8594 neonates of $<29$ weeks' GA were admitted to $\mathrm{CNN}$-participating NICUs during the study period. Of these, 570 neonates were excluded because they had major congenital anomalies (335) or received comfort care immediately after birth (235). Of the remaining 8024 eligible neonates, $2461(31 \%)$ did not receive surfactant, 3545 (44\%) received one dose of surfactant, and $2018(25 \%)$ received $>1$ dose of surfactant. From the available data on timing of surfactant, median age at first dose of surfactant was $1.6 \mathrm{~h}$ (IQR $0.7-3.8 \mathrm{~h}$ ), at second dose was $11.9 \mathrm{~h}$ (IQR $4.8-31.3 \mathrm{~h}$ ), at third dose was $33.5 \mathrm{~h}$ (IQR $15.1-58.3 \mathrm{~h}$ ), and at fourth dose was $37.6 \mathrm{~h}$ (IQR 17.1-95.2 h).

Among the 5563 neonates who received one or more doses of surfactant, the total number of doses received was 8220. These included 6407 doses of BLES (78\%), 1478 doses of beractant (18\%), 19 doses of poractant alfa $(0.2 \%)$, and 316 doses for which the type of surfactant was not documented (3.8\%). Of the 4449 neonates who received first dose of BLES, $32 \%$ received subsequent doses of BLES and only $0.4 \%$ received a different surfactant as subsequent doses. Of the 856 neonates who received a first dose of beractant, $44 \%$ received one dose, $48 \%$ received beractant as subsequent doses, and $8 \%$ received a different subsequent surfactant. Of the 15 neonates who received poractant alfa, $80 \%$ received only one dose and $20 \%$ received subsequent doses of poractant alfa. Overall, in total 1820 neonates received the same surfactant as in subsequent dose whereas 84 neonates received a different surfactant in subsequent doses.

\section{Baseline characteristics}

Baseline characteristics of the neonates are presented in Table 1. Compared to those who received one dose of surfactant or no surfactant, neonates who received multiple doses were more likely to be of younger GA, have lower BW, be delivered via cesarean section, and have higher rates of Apgar score $<5$ at $5 \mathrm{~min}$; and were less likely to have received a complete course of antenatal steroids. Rates of extensive resuscitation and pneumothorax were also higher in infants that required additional surfactant doses. Of note, timing of pneumothorax diagnosis in relation to surfactant administration was not available.

\section{Neonatal outcomes}

Neonatal outcomes for all three groups of infants are reported in Table 2. The composite outcome rate was higher among neonates who received 1 or $>1$ dose of surfactant, in a dose-dependent manner. Higher rates of mortality and major morbidities, such as severe neurological injury, BPD, and stage 3 or higher or treated ROP, were reported in the multiple surfactant dose group compared to the group that received one dose or none. Neonates who received additional surfactant doses also required longer periods of 
Table 1 Clinical characteristics and outcomes of the cohort.

\begin{tabular}{|c|c|c|c|c|c|}
\hline Variables & $\begin{array}{l}\text { No surfactant } \\
(n=2461)\end{array}$ & $\begin{array}{l}1 \text { dose of surfactant } \\
(n=3545)\end{array}$ & $\begin{array}{l}>1 \text { dose of surfactant } \\
(n=2018)\end{array}$ & $P$ value $^{\mathrm{a}}$ & $P$ value $^{\mathrm{b}}$ \\
\hline Maternal hypertension, no. (\%) & $249(10 \%)$ & $549(16 \%)$ & $404(21 \%)$ & $<0.01$ & $<0.01$ \\
\hline Maternal diabetes, no. (\%) & $282(12 \%)$ & $349(10 \%)$ & $190(10 \%)$ & 0.04 & 0.80 \\
\hline \multicolumn{6}{|l|}{ Antenatal steroid, no. $(\%)$} \\
\hline None & $168(7 \%)$ & $408(12 \%)$ & $293(15 \%)$ & \multirow[t]{3}{*}{$<0.01$} & \multirow[t]{3}{*}{$<0.01$} \\
\hline Partial & $413(17 \%)$ & $900(26 \%)$ & $533(27 \%)$ & & \\
\hline Complete & $1834(76 \%)$ & $2179(62 \%)$ & $1160(58 \%)$ & & \\
\hline Cesarean birth, no. (\%) & $1272(51 \%)$ & $2163(61 \%)$ & $1292(64 \%)$ & $<0.01$ & 0.02 \\
\hline Maternal chorioamnionitis, no. (\%) & $419(21 \%)$ & $564(20 \%)$ & $307(18 \%)$ & 0.33 & 0.29 \\
\hline Outborn, no. (\%) & $352(14 \%)$ & $567(16 \%)$ & $324(16 \%)$ & 0.16 & 0.95 \\
\hline Male sex, no. $(\%)$ & $1271(52 \%)$ & $1891(53 \%)$ & $1137(56 \%)$ & $<0.01$ & 0.03 \\
\hline Gestational age in weeks, mean (SD) & $26.8(1.4)$ & $26.0(1.6)$ & $25.4(1.6)$ & $<0.01$ & $<0.01$ \\
\hline Gestational age $22-25$ weeks, no. (\%) & $432(15 \%)$ & $1315(46 \%)$ & $1103(39 \%)$ & NA & NA \\
\hline Gestational age $26-28$ weeks, no. (\%) & $2022(39 \%)$ & $2230(43 \%)$ & $914(18 \%)$ & NA & NA \\
\hline Birth weight in g, mean (SD) & $1009(252)$ & $909(247)$ & $806(231)$ & $<0.01$ & $<0.01$ \\
\hline Small for gestational age, no. (\%) & $176(7 \%)$ & $278(8 \%)$ & $264(13 \%)$ & 0.35 & $<0.01$ \\
\hline Apgar score at $5 \mathrm{~min}<5$, no. $(\%)$ & $186(8 \%)$ & $646(18 \%)$ & $573(29 \%)$ & $<0.01$ & $<0.01$ \\
\hline $\begin{array}{l}\text { Extensive resuscitation (chest compression } \pm \\
\text { epinephrine), no. (\%) }\end{array}$ & $60(2 \%)$ & $230(7 \%)$ & $193(10 \%)$ & $<0.01$ & 0.01 \\
\hline Pneumothorax, no. (\%) & $27(1 \%)$ & $194(5 \%)$ & $231(11 \%)$ & $<0.01$ & $<0.01$ \\
\hline
\end{tabular}

$N A$ not applicable, $S D$ standard deviation.

${ }^{\text {a }} P$ value for comparison including all three groups.

${ }^{\mathrm{b}} P$ value for comparison of 1 dose of surfactant group vs $>1$ dose of surfactant group.

Table 2 Neonatal outcomes.

\begin{tabular}{|c|c|c|c|c|c|}
\hline Variables & $\begin{array}{l}\text { No surfactant } \\
(n=2461)\end{array}$ & $\begin{array}{l}1 \text { dose of surfactant } \\
(n=3545)\end{array}$ & $\begin{array}{l}>1 \text { dose of surfactant } \\
(n=2018)\end{array}$ & $P$ value $^{\mathrm{a}}$ & $P$ value $^{\mathrm{b}}$ \\
\hline Composite outcome, no. (\%) & $967(39 \%)$ & $2226(63 \%)$ & $1739(86 \%)$ & $<0.01$ & $<0.01$ \\
\hline Mortality, no. (\%) & $119(5 \%)$ & $463(13 \%)$ & $520(26 \%)$ & $<0.01$ & $<0.01$ \\
\hline Severe neurological injury, no. (\%) & $94(4 \%)$ & $350(10 \%)$ & $360(19 \%)$ & $<0.01$ & $<0.01$ \\
\hline BPD, no. (\%) & $795(35 \%)$ & $1632(53 \%)$ & $1167(76 \%)$ & $<0.01$ & $<0.01$ \\
\hline Mortality or BPD, no. (\%) & $907(37 \%)$ & $2076(59 \%)$ & $1649(82 \%)$ & $<0.01$ & $<0.01$ \\
\hline $\begin{array}{l}\text { Stage } 3 \text { or higher or treated retinopathy, } \\
\text { no. }(\%)\end{array}$ & $92(6 \%)$ & $355(14 \%)$ & $382(27 \%)$ & $<0.01$ & $<0.01$ \\
\hline $\begin{array}{l}\text { Length of invasive respiratory support, } \\
\text { median (IQR) }\end{array}$ & $0(0,0)$ & $7(2,23)$ & $20(6,41)$ & $<0.01$ & $<0.01$ \\
\hline $\begin{array}{l}\text { Length of noninvasive respiratory } \\
\text { support, median (IQR) }\end{array}$ & $34(14,48)$ & $38(23,53)$ & $38(7,57)$ & $<0.01$ & 0.22 \\
\hline $\begin{array}{l}\text { Length of supplemental oxygen use, } \\
\text { median (IQR) }\end{array}$ & $9(1,34)$ & $33(9,65)$ & $58(17,97)$ & $<0.01$ & $<0.01$ \\
\hline
\end{tabular}

Composite outcome included mortality prior to discharge or severe neurological injury or BPD.

$B P D$ bronchopulmonary dysplasia, $I Q R$ interquartile range.

${ }^{a} P$ value for comparison including all three groups.

${ }^{\mathrm{b}} P$ value for comparison of 1 dose of surfactant group vs $>1$ dose of surfactant group.

invasive respiratory support and supplemental oxygen than those who received one dose or none. The period of noninvasive respiratory support was significantly longer in the multiple dose group when comparing all three groups $(P<$
0.01); however, there was no significant difference between the single and multiple dose groups $(P=0.22)$.

Results of multiple logistic regression analyses adjusted for sex, GA, SGA, cesarean section, maternal hypertension, 


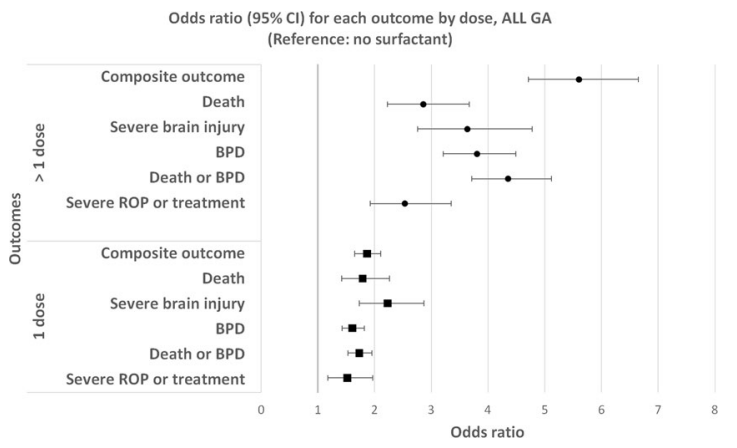

Fig. 1 Multivariable analyses: adjusted odds ratios (95\% CI). BPD bronchopulmonary dysplasia, CI confidence interval, GA gestational age, ROP retinopathy of prematurity. Composite outcome included mortality prior to discharge or severe neurological injury or BPD or stage 3 or higher or treated retinopathy.

receipt of antenatal steroids, Apgar score at $5 \mathrm{~min}$ of $<5$, and extensive resuscitation are reported in Fig. 1 (Supplementary Table 1). Generalized estimating equation was applied in each regression model to account for the correlation within the same site and within multiple births. Using the no surfactant group as a reference, the odds of the composite outcome and the individual components of the composite outcome were significantly higher among neonates who received multiple doses of surfactant vs those who received a single dose of surfactant; and higher among those who received a single dose of surfactant vs those who did not receive surfactant. This pattern of increases in odds reflected a dosedependent effect.

Since GA is a very important determinant of outcomes, we conducted subgroup analyses to compare outcomes within two GA subgroups: 22-25 weeks' GA and 26-28 weeks' GA (Fig. 2, Supplementary Table 2). The results were similar to those identified in the primary analyses. Comparing neonates who received multiple surfactant doses of the same surfactant vs different surfactants, differences were not statistically significant (data not shown).

\section{Discussion}

In this large, multicenter, contemporaneous cohort study, we identified that, among neonates of $<29$ weeks' gestation, the following characteristics were associated with receipt of multiple doses of surfactant: maternal hypertension, lack or incomplete doses of antenatal steroids, cesarean birth, male sex, younger GA, lower BW, and need for extensive cardiopulmonary resuscitation. Neonates who received one or multiple doses of surfactant had higher odds of mortality and major morbidities-specifically, severe neurological injury, BPD, and stage 3 or higher ROP - than those who did not receive surfactant. The associations were identified to be dose dependent.
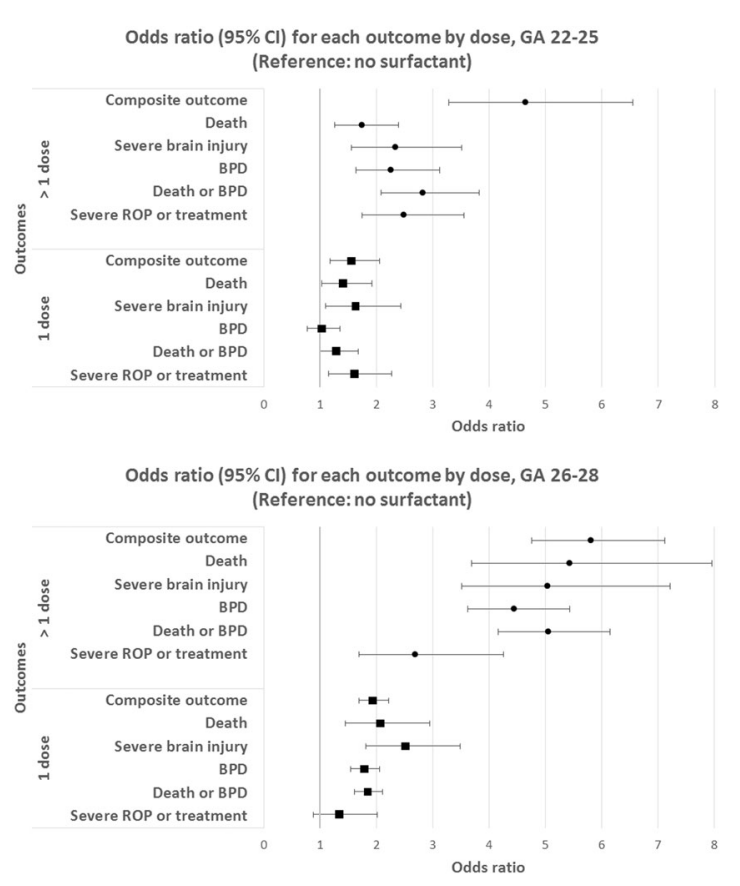

Fig. 2 Subgroup analyses for 2 gestational age groups: adjusted odds ratios $(95 \%$ CI). BPD bronchopulmonary dysplasia, CI confidence interval, GA gestational age, ROP retinopathy of prematurity. Composite outcome included mortality prior to discharge or severe neurological injury or BPD or stage 3 or higher or treated retinopathy.

Concordant with our findings, lower GA and BW have been suggested as risk factors for receipt of multiple doses of surfactant $[10,11,14]$. Tsakaldis et al. compared 28 neonates of $<32$ weeks' GA who received multiple doses of surfactant with 98 who only received a single dose. They reported that neonates in the multiple dose group had higher rates of RDS within $6 \mathrm{~h}$ after birth compared to the single dose group [14]. They also reported a higher rate of maternal chorioamnionitis in the group who received multiple doses. Herting et al. [12] reported that chorioamnionitis and group B streptococcal infection may lead to surfactant inactivation due to inflammatory mediators and increase the severity of RDS. We did not identify such an association, possibly due to a lack of uniformity for the diagnosis of chorioamnionitis. Katz et al. [11] studied neonates of $\mathrm{BW}<1000 \mathrm{~g}$ for the development of atelectasis after initial surfactant treatment for RDS. Of 165 eligible neonates, 39 received no surfactant (mean GA 27.7 weeks, mean BW $856 \mathrm{~g}$ ); 116 received a single dose of surfactant (mean GA 26 weeks, mean BW $751 \mathrm{~g}$ ); and 25 received surfactant after 6 days of initial therapy due to atelectasis (termed "post surfactant slump") (mean GA 24.7 weeks, mean BW $647 \mathrm{~g}$ ). The observations of lower GA and BW in that study were similar to our observations for repeated doses in the current study. Katz et al. [11] also identified a lack of antenatal steroids as a risk factor for requiring multiple doses of surfactant. Previous randomized studies of 
surfactant therapy have reported repeat surfactant administration rates between 20 and $50 \%$ [4]. A previous study comparing BLES and poractant alfa reported that $20 \%$ of patients received a second dose of surfactant [22]. In our cohort, $36 \%$ of patients received a second dose of surfactant among those who received any dose of surfactant. This is compatible with the literature considering the differences in population characteristics between randomized and pragmatic settings. The threshold for intubation of a preterm neonate who needs distending pressure and oxygen to maintain oxygenation varies not only between countries but also between units within countries [23]. Overall, these findings support the concept that multiple surfactant doses could potentially reflect severe underlying respiratory immaturity and challenges associated with gas exchange in these extremely preterm neonates.

Neonates who received multiple doses of surfactant were identified to have increased odds of mortality and major morbidities in a dose-dependent manner. It is possible that receipt of multiple surfactant doses is a marker of disease severity not captured by statistical adjustment. Our subgroup analyses of neonates 22-25 weeks' GA and 26-28 weeks' GA confirmed the results. Numerically (not statistically), the odds of adverse outcomes were higher in those 26-28 weeks' GA compared to those $<26$ weeks' GA. This may have been due to outcomes being more prevalent in the lower compared to the higher GA group; however, it reinforces our point that it is the severity of lung disease that necessitates repeated doses of surfactant and not that surfactant is harmful. This concept creates an important avenue for identification of at-risk neonates based on need for or administration of more than one dose of surfactant and quality improvement initiatives for primary, secondary, and tertiary prevention. For primary prevention, it stresses the need for a complete and timely course of antenatal steroids. For secondary prevention, it creates an opportunity to explore what criteria should be used to administer surfactant such that atelectrauma and barotrauma or oxytrauma to immature lungs are minimized. Both primary and secondary prevention strategies are applicable to any preterm neonate and not specially targeted to preterm neonates who need additional doses of surfactant. For tertiary prevention, it alerts clinicians to potential underlying lung immaturity and allows them to manage such neonates carefully so that ventilator-induced injury can be minimized during their further course in the NICU. In addition, it alerts clinicians to the potential for other complications for which neonates are at higher risk and suggests they deploy preventive strategies aggressively (e.g., oxygen administration control or saturation target control for prevention of ROP or prevention of hypercarbia, which is associated with brain injury [24]).

The idea of giving multiple doses of surfactant came about in the 1990s when the benefits of surfactant treatment were realized and it was hypothesized that prophylactic administration of multiple doses may be more beneficial. In a randomized trial, Corbet et al. [25] reported lower mortality and lower rates of necrotizing enterocolitis in a group of infants who received three prophylactic doses of surfactant vs one prophylactic dose of surfactant. In a Cochrane review of three randomized trials, Soll and Ozek identified lower risks of pneumothorax and mortality associated with multiple doses of surfactant [1]. However, these studies were done in the era of low use of prenatal steroids and higher use of invasive ventilation. The current practice, which includes high use of antenatal steroids and avoidance of mechanical ventilation strategies when possible, has prompted several units to practice minimally invasive surfactant administration. Thus, our data from recent years provide information that can be used for several purposes at both individual and unit levels to target neonates at risk of severe neonatal outcomes.

Major strengths of our study are that it was a large, contemporaneous, multicenter study of $>8000$ neonates that used a reliable and accurate population-wide database and included a detailed evaluation of risk factors associated with receiving multiple doses of surfactant. However, there were some limitations. First, data on the severity of RDS were not collected. We electively did not report data on RDS as the definition and diagnosis of RDS in the era of prophylactic surfactant administration are controversial at best. Second, we did not have data on respiratory parameters prior to the administration of surfactant; thus, the respiratory statuses for initial or subsequent surfactant treatments were unknown. The criteria for surfactant administration may have varied between participating units and the threshold levels (usually between $\mathrm{FiO}_{2}$ 30-40\%) may have differed. However, we have identified that these differences were not substantial in Canada [23]. Third, the majority of infants received BLES as surfactant; however, some received beractant or poractant alfa during the study period. A few neonates received two different types of surfactant. We did not analyze the data by type of surfactant as our main focus was to identify outcome differences according to number of doses and not type of surfactant. Fourth, it is possible that repeat doses of surfactant may have been given for hypoxemia associated with pulmonary hypertension in the immediate period and not to treat respiratory immaturity. We did not collect information regarding the reasons for repeat surfactant doses. Finally, in the later years of the study, some neonates may have received surfactant via techniques other than endotracheal intubation, and we do not know whether or not this necessitated repeated doses of surfactant. Our database did not include information on method of surfactant administration.

In conclusion, among infants of $<29$ weeks' gestation, those who received single or multiple doses of surfactant 
had higher odds of mortality and major morbidities, including severe brain injury, BPD, and severe retinopathy, than those who did not receive surfactant. This finding suggests that receipt of multiple surfactant doses could potentially be a useful marker for severe underlying respiratory immaturity and could be used to identify patients who should be closely monitored to prevent ongoing lung injury and other adverse outcomes.

\section{Data availability}

The data sets generated and analyzed during the current study are with the corresponding author but current data transfer approval agreements do not allow for data to be made available to others.

Acknowledgements The authors thank all site investigators and data abstractors of the CNN and the Canadian Preterm Birth Network (CPTBN). A full list of Network member investigators and their affiliations appears in Supplementary Information. We thank Heather McDonald Kinkaid, $\mathrm{PhD}$, of the Maternal-infant Care Research Centre (MiCare) at Mount Sinai Hospital in Toronto, ON, Canada, for editorial support in preparing this manuscript; and other MiCare staff, for organizational support.

Funding This study was supported by a grant from the Canadian Institutes of Health Research (CIHR) funding the Canadian Preterm Birth Network (PBN 150642). Organizational support for the CNN and the Canadian Preterm Birth Network was provided by the Maternal-infant Care Research Centre (MiCare) at Mount Sinai Hospital in Toronto, ON, Canada. MiCare is supported by a CIHR Team Grant (CTP 87518), the Ontario Ministry of Health and Long-Term Care, and the participating hospitals. PSS holds a CIHR Applied Research Chair in Reproductive and Child Health Services and Policy Research (APR-126340).

Author contributions HC and PSS conceptualized and designed the study, drafted the initial manuscript, and reviewed and revised the manuscript. AM, BL, EHN, RA, GE, and MB participated in study design and data interpretation and reviewed and revised the manuscript. EWY analyzed the data and reviewed and revised the manuscript. All authors approved the final manuscript as submitted and agree to be accountable for all aspects of the work in ensuring that questions related to its accuracy or integrity are appropriately investigated and resolved.

\section{Compliance with ethical standards}

Conflict of interest The authors declare that they have no conflict of interest.

Publisher's note Springer Nature remains neutral with regard to jurisdictional claims in published maps and institutional affiliations.

\section{References}

1. Soll R, Ozek E. Multiple versus single doses of exogenous surfactant for the prevention or treatment of neonatal respiratory distress syndrome. Cochrane Database Syst Rev. 2009;21:CD000141.
2. Been JV, Rours IG, Kornelisse RF, Jonkers F, de Krijger RR, Zimmermann LJ. Chorioamnionitis alters the response to surfactant in preterm infants. J Pediatr. 2010;156:10-15.e1.

3. Horbar JD, Soll RF, Sutherland JM, Kotagal U, Philip AG, Kessler DL, et al. A multicenter randomized, placebo-controlled trial of surfactant therapy for respiratory distress syndrome. N Engl J Med. 1989;320:959-65.

4. Singh N, Halliday HL, Stevens TP, Suresh G, Soll R, Rojas-Reyes MX. Comparison of animal-derived surfactants for the prevention and treatment of respiratory distress syndrome in preterm infants. Cochrane Database Syst Rev. 2015;12:CD010249.

5. Rojas-Reyes MX, Morley CJ, Soll R. Prophylactic versus selective use of surfactant in preventing morbidity and mortality in preterm infants. Cochrane Database Syst Rev. 2012;3:CD000510.

6. Bahadue FL, Soll R. Early versus delayed selective surfactant treatment for neonatal respiratory distress syndrome. Cochrane Database Syst Rev. 2012;11:CD001456.

7. Subramaniam P, Ho JJ, Davis PG. Prophylactic nasal continuous positive airway pressure for preventing morbidity and mortality in very preterm infants. Cochrane Database Syst Rev. 2016;6: CD001243.

8. Stevens TP, Harrington EW, Blennow M, Soll RF. Early surfactant administration with brief ventilation vs. selective surfactant and continued mechanical ventilation for preterm infants with or at risk for respiratory distress syndrome. Cochrane Database Syst Rev. 2007;4:CD003063.

9. More K, Sakhuja P, Shah PS. Minimally invasive surfactant administration in preterm infants: a meta-narrative review. JAMA Pediatr. 2014;168:901-8.

10. Speer CP, Robertson B, Curstedt T, Halliday HL, Compagnone D, Gefeller O, et al. Randomized European multicenter trial of surfactant replacement therapy for severe neonatal respiratory distress syndrome: single versus multiple doses of Curosurf. Pediatrics. 1992;89:13-20.

11. Katz LA, Klein JM. Repeat surfactant therapy for postsurfactant slump. J Perinatol. 2006;26:414-22.

12. Herting E, Gefeller O, Land M, Sonderen LV, Harms K, Robertson B, et al. Surfactant treatment of neonates with group B streptococcal infection. Pediatrics. 2000;106:957-64.

13. Kattwinkel J, Bloom BT, Delmore P, Glick C, Brown D, Lopez S, et al. High-versus low-threshold surfactant retreatment for neonatal respiratory distress syndrome. Pediatrics. 2000;106:282-8.

14. Tsakaldis C, Giougki E, Karagianni P, Dokos C, Rallis D, Nikolaidis $\mathrm{N}$. Is there a necessity for multiple doses of surfactant for respiratory distress syndrome of premature infants? Turk J Pediatr. 2015;54:368-75.

15. Hoekstra RE, Jackson JC, Myers TF, Frantz ID 3rd, Stern ME, Powers WF, et al. Improved neonatal survival following multiple doses of bovine surfactant in very premature neonates at risk for respiratory distress syndrome. Pediatrics. 1991;88:10-18.

16. Dunn MS, Shennan AT, Possmayer F. Single- versus multipledose surfactant replacement therapy in neonates of 30 to 36 weeks' gestation with respiratory distress syndrome. Pediatrics. 1990;84:564-71.

17. Lee SK, Beltempo M, McMillan DD, Seshia M, Singhal N, Dow $\mathrm{K}$, et al. Outcomes and care practices for preterm infants born at less than 33 weeks' gestation: a quality-improvement study. CMAJ. 2020;192:E81-91.

18. The Canadian Neonatal Network. The Canadian Neonatal Network Abstractor's Manual v.3.4.1. The Canadian Neonatal Network; 2019. http://www.canadianneonatalnetwork.org/portal/ CNNHome/Publications.aspx.

19. Shah PS, Seidlitz W, Chan P, Yeh S, Musrap N, Lee SK, et al. Internal audit of the Canadian Neonatal Network data collection system. Am J Perinatol. 2017;34:1241-9. 
20. Davis DJ, Barrington KJ. Recommendations for neonatal surfactant therapy. Pediatr Child Health. 2005;10:109-16.

21. Papile LA, Burstein J, Burstein R, Koffler H. Incidence and evolution of subependymal and intraventricular hemorrhage: a study of infants with birth weights less than $1,500 \mathrm{~g}$. J Pediatr. 1978;92:529-34.

22. Lemyre B, Fusch C, Schmölzer GM, Bouali RN, Reddy D, Barrowman $\mathrm{N}$, et al. Poractant alfa versus bovine lipid extract surfactant for infants $24+0$ to $31+6$ weeks gestational age: A randomized controlled trial. PLoS ONE. 2017;12:e0175922.
23. Beltempo M, Isayama T, Vento M, Lui K, Kusuda S, Lehtonen L, et al. Respiratory management of extremely preterm infants: an international survey. Neonatology. 2018;114:28-36.

24. Bel FV, Vaes J, Groenedaal F. Prevention, reduction and repair of brain injury of the preterm infant. Front Physiol. 2019;10:181.

25. Corbet A, Gerdes J, Long W, Avila E, Puri A, Rosenberg A, et al. Double-blind, randomized trial of one versus three prophylactic doses of synthetic surfactant in 826 neonates weighing 700 to 1100 grams: effects on mortality rate. American Exosurf Neonatal Study Groups I and IIa. J Pediatr. 1995;126:969-78. 